\title{
ESTUDO INVESTIGATIVO DOS EFEITOS SEDATIVO E ANTINOCICEPTIVO DA CLONIDINA E DA RILMENIDINA EM EQUINOS
}

(Investigative study of sedative and antinociceptive effects of clonidine and rilmenidine in horses)

\author{
José Edgard de Oliveira Alves ${ }^{1}$, Scarlath Ohana Penna dos Santos, Evelyn Mayara Perrut \\ Vieira, Fábio Sartori, Marcelo Flores Catelli
}

${ }^{1}$ Correspondência: edgardoliveiraalves@hotmail.com

\begin{abstract}
RESUMO: Clonidina e rilmenidina são fármacos que possuem diversos efeitos sistêmicos em função de suas ações sobre os receptores alfa-adrenérgicos e imidazolínicos, tais como hipotensão, bradicardia, redução do débito cardíaco e da resistência vascular periférica e sedação e antinocicepção dose-dependentes. O presente estudo teve como objetivo investigar e comparar os efeitos farmacológicos da clonidina e da rilmenidina sobre o limiar nociceptivo e a sedação de equinos. Foram utilizados 6 equinos adultos, machos e fêmeas, os quais foram submetidos a três tratamentos: (1) controle; (2) clonidina 0,002 mg/kg; e (3) rilmenidina $0,021 \mathrm{mg} / \mathrm{kg}$. Os fármacos foram administrados por via oral e os animais foram avaliados em três momentos, sendo o primeiro (T0) imediatamente antes da administração dos medicamentos, após 60 minutos (T60) e após 120 minutos (T120). Foram investigados os efeitos sedativo e antinociceptivo através dos modelos de abaixamento de cabeça e da latência para o reflexo de retirada do membro, respectivamente. Nossos ensaios apontaram abaixamento de cabeça com significância estatística no tratamento com clonidina, indicando um efeito sedativo, o que não ocorreu com a administração de rilmenidina, e ambos os fármacos não foram capazes de aumentar o limiar nociceptivo. Concluímos que a clonidina por via oral é capaz de induzir leve sedação na dose testada neste estudo e que as doses utilizadas tanto para clonidina quanto para a rilmenidina não produzem antinocicepção em equinos.
\end{abstract}

Palavras-chave: agonistas alfa2-adrenérgicos, imidazolinas, nocicepção, analgesia, farmacologia clínica

\begin{abstract}
Clonidine and rilmenidine are drugs that have several systemic effects due its actions on alpha-adrenergic and imidazoline receptors, such as hypotension, bradycardia, reduction of the cardiac output and peripheral vascular resistance and dose-dependent sedation and antinociception. This study aims to investigate and compare the pharmacological effects of clonidine and rilmenidine over the nociceptive threshold and the sedation in horses. Were used six adult horses, males and females, that were submitted to three treatments: (1) Control; (2) clonidine 0,002 mg/kg; and (3) rilmenidine 0,021 mg/kg. The drugs were administered by oral rote and the animals were assessed in three moments, therefore the first time (T0) immediately before drugs administration, after 60 minutes (T60) and after 120 minutes (T120). Were investigate the sedative and antinociceptive effects by head ptosis and latency for limb withdrawal reflex methods, respectively. Ours tests showed head ptosis with statistical significance in treatment with clonidine, indicating a sedative effect, which do not occurs with rilmenidine administration, and both drugs were not able to improve the nociceptive threshold. Conclude that clonidine is able to induce mild sedation on tested dose by oral rote administration and which the used doses both for clonidine as rilmenidine in this study do not produce antinociception in horses.
\end{abstract}

Key Words: alfa2-adrenoceptor agonists, imidazolines, nociception, analgesia, clinical pharmacology 


\section{INTRODUÇÃO}

A clonidina é um dos fármacos alfa2-agonistas mais amplamente investigados na anestesiologia e na terapêutica da dor (GIOVANNONI et al., 2009). Foi sintetizada na Alemanha em meados da década de 1960 com o objetivo de se obter um medicamento que atuasse perifericamente em receptores adrenérgicos visando um efeito descongestionante nasal através da administração em gotas. Contudo, apesar do efeito vasoconstritor e descongestionante em baixas concentrações da substância, foram descobertos posteriormente outros efeitos mais marcantes, tais como hipotensor, bradicárdico e antisialogogo. Este primeiro fez que com a clonidina fosse posteriormente introduzida na rotina clínica como agente anti-hipertensivo (STÄHLE, 2000). Dentre seus efeitos adversos, seu importante efeito sedativo, acompanhado de hipnose e analgesia, possibilitou que este fármaco pudesse ser introduzido na prática anestesiológica, sendo aplicada como medicação pré-anestésica e em anestesias raquidianas em humanos (ALVES, 2004) e em anestesias epidurais em equinos (GUIRRO et al., 2011) e cães (BRONDANI et al., 2004) e como sedativo e ansiolítico em pequenos animais (CARVALHO et al., 2013; OGATA; DODMAN, 2011).

Considera-se que o mecanismo de ação da clonidina consista na estimulação de receptores alfa2adrenérgicos situados no sistema nervoso central, inibindo da liberação de noradrenalina nas terminações nervosas adrenérgicas pré-sinápticas (SPINOSA; SPINOSA, 1991). O efeito antinociceptivo da clonidina é obtido através de sua ação em diferentes regiões do sistema nervoso central, que incluem o núcleo dorsal da rafe, a área cinzenta periaquedutal e o locus coeruleous. Considera-se, ainda, a sua ação em sítios periféricos, supraespinhais e espinhais e a ativação de receptores alfa2-adrenérgicos póssinápticos das vias descendentes noradrenérgicas e dos neurônios colinérgicos e liberação de óxido nítrico e substância tipo encefalina (ALVES, 2004). O uso oral da clonidina tem sido estudado em alguns trabalhos, onde observou-se que o pré-tratamento com administração oral de clonidina antes da canulação venosa permite reduzir a ansiedade e a dor e promove a estabilidade cardiovascular nos pacientes. (GIOVANNONI et al., 2009).

A rilmenidina é um fármacos antihipertensivo que exerce sua ação através de um efeito simpatolítico no centro vasomotor localizado no bulbo raquidiano (ONRAT et al., 2004). O mecanismo farmacológico central da rilmenidina é análogo ao da clonidina, isto é, há estimulação dos receptores imidazolínicos no núcleo reticular lateral (FELDMAN, 1990). Apesar disso, a rilmenidina tem 2 vezes mais afinidade que a clonidina para receptores imidazolínicos (BRICCA et al., 1989). A rilmenidina, ao se ligar aos receptores $I_{1}$ no núcleo reticular lateral do tronco cerebral, provoca uma redução sistêmica do tônus simpático, que, por sua vez, leva à uma redução da resistência vascular periférica, contribuindo para a redução da pressão arterial. Ocorre também uma simpatoinibição em nível renal, por conta da presença de receptores imidazolínicos neste órgão (REID, 2000). Estudos experimentais tem demonstrado que a administração de rilmenidina por via oral ou intravenosa em ratos hipertensos reduz o tônus simpático, a frequência cardíaca e a pressão arterial (ONRAT et al., 2004). Seu efeito antinociceptivo já foi descrito por diversos autores em modelos de dor experimental utilizando animais de laboratório (SABETKASAIE et al., 2007; HAJHASHEMI et al., 2007; 
ROMAN, 2004; AZEVEDO, 2002), permitindo, inclusive, vislumbrar um fármaco com potencial efeito analgésico e com menores efeitos adversos em relação à clonidina (SABETKASAIE et al., 2007; AZEVEDO, 2002).

Tendo em consideração a importância do controle da dor e da busca por novos fármacos mais eficientes e com menores efeitos adversos para o manejo da mesma, o presente estudo tem como objetivo investigar e comparar os efeitos sedativo e antinociceptivo com a administração de $0,002 \mathrm{mg} / \mathrm{kg}$ de clonidina ou $0,021 \mathrm{mg} / \mathrm{kg}$ de rilmenidina em equinos, uma vez que, até o momento, inexistem estudos na literatura mundial avaliando os efeitos da rilmenidina nesta espécie e o estudos abordando os efeitos da clonidina por via oral ainda são escassos em equinos.

\section{MATERIAL E MÉTODOS}

O experimento foi realizado na Fazenda Experimental da Universidade Severino Sombra, utilizando 6 equinos, machos e fêmeas adultos, sem raça definida, criados em regime extensivo e pesando entre 280 e $440 \mathrm{~kg}$, os quais foram submetidos a três tratamentos diferentes, sendo eles: CON (Controle); CLO $\left(0,002 \mathrm{mg} / \mathrm{kg}\right.$ de clonidina $\left.{ }^{1}\right) ;$ e RIL $\left(0,021 \mathrm{mg} / \mathrm{kg}\right.$ de rilmenidina $\left.{ }^{2}\right)$. Os fármacos foram administrados em comprimidos por via oral junto a $100 \mathrm{~g}$ de ração, exceto para o tratamento controle, que recebeu a mesma quantidade de ração sem a presença dos comprimidos. Para garantir total depuração dos fármacos do organismo dos animais entre os experimentos, foi preconizado um período de wash out de sete meias-vidas de eliminação dos

\footnotetext{
${ }^{1}$ Atensina ${ }^{\circledR} 0,2 \mathrm{mg}$, Boehringer Ingelhein do Brasil Quím. e Farm. Ltda., São Paulo, SP, Brasil.

${ }^{2}$ Hyperium ${ }^{\circledR} 1 \mathrm{mg}$, Laboratórios Servier do Brasil Ltda., Rio de Janeiro, RJ, França.
}

fármacos para que os animais pudessem ser submetidos ao tratamento seguinte. As avaliações foram feitas imediatamente antes da administração dos fármacos (T0) e subsequentemente em 60 (T60) e 120 (T120) minutos após a administração oral dos medicamentos.

A sedação foi avaliada através do método de abaixamento da cabeça (AC) (KAMERLING et al., 1988), o qual consiste na mensuração da altura em metros do chão ao lábio inferior do animal através do uso de uma régua graduada. Para fins estatísticos e levando-se em conta as eventuais diferenças de altura individuais de cada animal, a variação da $A C$ nos tempos avaliados foi expressa em percentual em relação à $A C$ do próprio animal em T0.

A determinação dos efeitos dos fármacos sobre 0 limiar nociceptivo cutâneo foi realizada através do uso de um aparato adaptado do método descrito por Kamerling et al. (1985), o qual utiliza a emissão de um foco de luz sobre a pele dos animais e quantifica 0 efeito antinociceptivo através do tempo de latência para a resposta ao estímulo térmico por movimento de esquiva. Seguindo este princípio, utilizamos uma lâmpada infravermelha de $250 \mathrm{~W}$ e $220 \mathrm{~V}^{3}$, emitindo um estímulo nociceptivo regular para todos os animais. A região escolhida para incidência da luz foi a face lateral do boleto anterior direito, aplicando-se o teste do tempo de latência para o reflexo de retirada do membro (LRRM). A região exposta à luz foi tricotomizada e pintada com tinta preta lavável, de modo a favorecer uma reflexão uniforme de luz e, consequentemente, absorção igualitária de calor nos tecidos com diferentes pigmentações.

Após formatação e tabulação dos dados, os resultados foram expressos

\footnotetext{
${ }^{3}$ EMPALUX ${ }^{\circledR}$, modelo IV22514. Empalux, São José dos Pinhais, Paraná, Brasil.
} 
como a média \pm o erro padrão da média (EPM) para cada tratamento realizado e foram analisados por análise de variância (ANOVA), adotando-se Newman-Keuls Multiple Comparison Test como pós teste, com grau de confiança de $95 \%(p<0,05)$. Todos os cálculos e gráficos foram realizados com software de computador ${ }^{4}$. As avaliações foram realizadas por um mesmo observador em todos os animais, de modo a minimizar falhas de interpretação. Toda a metodologia foi aprovada pela Comissão de Ética no Uso de Animais da Universidade Severino Sombra (001/2014).

\section{RESULTADOS E DISCUSSÃO}

O abaixamento de cabeça provocado pela clonidina de $-12,27 \pm 4,0 \%$ e $-11,88$ $\pm 4,5 \%$ após 60 e 120 minutos, respectivamente, indica a ocorrência de algum grau de sedação, o que não ocorreu com a rilmenidina (Gráfico 1), ainda que tenha sido observado aumento na frequência de bocejos nos animais no tratamento com rilmenidina, - que nos leva a acreditar que a rilmenidina induza algum grau de sonolência nas doses testadas neste estudo, mas sem, no entanto, se constatar a ocorrência de sedação pela quantificação do abaixamento de cabeça.

Gráfico 1 - Percentuais de variação da AC nos tempos 60 e 120 minutos em relação à avaliação basal.

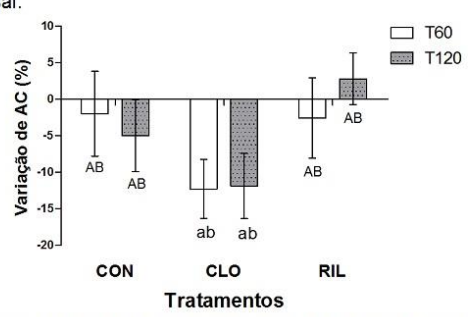

AC: Altura da cabeça; T60: Avaliação após 60 minutos; T120: Avaliação após 120 minutos; CON Controle; CLO: Clonidina $0,002 \mathrm{mg} / \mathrm{kg}$; RIL: Rilmenidina $0,021 \mathrm{mg} / \mathrm{kg}$; A: Resultados não difere estatisticamente entre os tratamentos no mesmo momento de avaliaçấ; a : Resultados difere estatisticamente dentro do mesmotratamento em relação à T0; b: Resultados diferem estatisticamente entre os tratamentos no mesmo momento de avaliaçẫo.

\footnotetext{
${ }^{4}$ GraphPad Prism 5. GraphPad Software, Inc.,
} San Diego, CA, USA.
Uma explicação para este resultado é o efeito sedativo de menor intensidade que a rilmenidina possui em relação à clonidina, haja vista o fato de a primeira ser duas vezes mais seletiva para a região do bulbo rostroventrolateral que para o locus coeruleus (TIBIRIÇÁ et al., 1991), região do sistema nervoso central responsável pelo controle do ciclo sono-vigília e sítio onde se encontram predominantemente receptores alfa2-adrenérgicos.

Ambos os fármacos testados não foram capazes de aumentar o tempo de latência para uma resposta ao estímulo nociceptivo com significância estatística Contudo, a dose de $0,002 \mathrm{mg} / \mathrm{kg}$ de clonidina elevou em 2 segundos o valor absoluto do tempo de LRRM, assim como a rilmenidina que aumentou este $10 \pm 1$ segundos na avaliação basal para $11 \pm 2$ segundos ao final dos 120 minutos, respectivamente. Como já dito, estes valores não representam diferenças estatísticas, mas podem indicar uma tendência ao aumento da LRRM como efeito farmacológico, que poderia ser conclusivo com um aumento das doses utilizadas (Tabela 1).

\begin{tabular}{l} 
Tabela 1 - LRRM dos tratamentos propostos nos tempos T0, T60 e T120. \\
\cline { 2 - 4 } \\
\cline { 2 - 4 } \\
\cline { 2 - 4 }
\end{tabular}

Apesar de diversos autores afirmarem que a rilmenidina produz antinocicepção (SABETKASAIE et al., 2007; HAJHASHEMI et al., 2007; ROMAN, 2004; AZEVEDO, 2002), o mecanismo de ação pelo qual a antinocicepção parece ser produzida ainda não está completamente esclarecido. Azevedo (2002) atribui o efeito antinociceptivo à ação sobre os receptores alfa-adrenérgicos, enquanto Sabetkasaie et al. (2007) propõem uma via que envolve o receptor NMDA participando deste efeito farmacológico. 
Contrariando estas informações, não observamos efeito antinociceptivo em nossos ensaios, fato este que pode ser atribuído à dose utilizada, uma vez que o efeito é dose-dependente. Como inexistem até 0 momento estudos utilizando a rilmenidina em equinos até o momento na literatura mundial, ainda não haviam doses que pudessem ser referenciadas. Entretanto, sabendo-se que os efeitos farmacológicos da rilmenidina são dose-dependentes, adotamos em nossa linha de pesquisa os valores de três vezes a dose usualmente aplicada na terapêutica antihipertensiva em humanos, extrapolados para a espécie equina por meio de cálculo alométrico (PACHALY, 2006). Todavia, estes valores podem ainda não ter sido suficientes para a obtenção de um efeito clínico mais evidente.

A literatura com relação à administração de clonidina em equinos é escassa no que se refere à utilização da via oral. Entretanto, existem diversos trabalhos avaliando seus efeitos analgésicos quando administrada por via epidural, tanto em equinos quanto em outras espécies (GUIRRO et al., 2011; DÓRIA et al., 2008; BRONDANI et al., 2004). De uma maneira geral, estes autores concluem que a administração epidural de clonidina produz analgesia intraoperatória e póscirúrgica satisfatórias. Em macacos, estudos anteriores avaliando três agonistas alfa2-adrenérgicos (clonidina, dexmedetomidina e xilazina) não foram capazes de separar os efeitos sedativos e analgésicos entre as drogas testadas. Neste ensaio, foi relatada a ocorrência de efeito antinociceptivo em todos os fármacos, contudo, este só ocorreu com doses que provocaram simultaneamente outros efeitos fisiológicos e comportamentais

WOODS, 1993). Partindo deste princípio, a ausência da sedação pela rilmenidina na dose utilizada constatada em nossos resultados pode ter reduzido um possível efeito antinociceptivo deste medicamento. Do mesmo modo, pressupõe-se que um grau maior de sedação provocado pela clonidina poderia ser acompanhado de um efeito antinociceptivo evidente.

Cabe ressaltar que, apesar da não observação dos efeitos esperados sobre - limiar nociceptivo, ainda não se pode descartar a hipótese considerada neste experimento de que clonidina e rilmenidina possam produzir sedação e antinocicepção dose-dependentes em equinos, uma vez que a administração oral influencia na biodisponibilidade e, consequentemente, no efeito dos fármacos. Olson et al. (2015) observaram redução significante da dor e da inflamação utilizando a suspensão oral de meloxicam em equinos. Estes autores destacam, ainda, a administração oral do fármaco como um método prático e seguro para o controle da dor e da inflamação pós-castração em cavalos. Recentemente, estudos avaliando os efeitos do cloridrato de sotalol, um beta-bloqueador adrenérgico não-seletivo, após administração oral, apontaram biodisponibilidade de $48 \%$, o que, para os autores, foi considerado um resultado intermediário, porém promissor em função da facilidade de administração e dos índices de biodisponibilidade encontrados (BROUX et al., 2015). Serrano-Rodríguez et al. (2015), investigando os efeitos do ramipril em equinos, um inibidor da enzina conversora de angiotensina (ECA), destacam que, apesar da biodisponibilidade baixa após administração oral, altas doses do fármaco possuem absorção entérica e bioconversão suficientes para produzir inibição de até $85 \%$ da concentração sérica da ECA. Sabendo-se, então, que a biodisponibilidade após administração oral está relacionada com a dose administrada, maiores estudos avaliando os efeitos de ambos os fármacos em diferentes doses poderão 
ajudar a elucidar os questionamentos ainda não respondidos neste estudo com relação à viabilidade da aplicação destes fármacos no tratamento da dor ou como adjuvantes em protocolos de controle da dor em equinos.

\section{CONCLUSÃO}

Conclui-se que a dose de 0,002 $\mathrm{mg} / \mathrm{kg}$ é capaz de induzir sedação em equinos, efeito que não ocorre com a dose de $0,021 \mathrm{mg} / \mathrm{kg}$ de rilmenidina e ambos os fármacos não são capazes de produzir antinocicepção nas doses testadas. Todavia, novos estudos avaliando doses mais altas de ambos os fármacos podem contribuir futuramente para um melhor entendimento dos efeitos farmacológicos da clonidina e da rilmenidina na espécie equina.

\section{AGRADECIMENTOS}

Os autores agradecem à USS/FUSVE pela bolsa de iniciação científica concedida para realização deste estudo.

\section{NOTAS INFORMATIVAS}

Toda a metodologia foi aprovada pela Comissão de Ética no Uso de Animais da Universidade Severino Sombra (001/2014).

\section{REFERÊNCIAS}

ALVES, T. C. A. Clonidina. In: CAVALCANTI, I. L.; CANTINHO, F. A. F.; VINAGRE, R. C. O. Anestesia Venosa. Rio de Janeiro: Sociedade de Anestesiologia do Estado do Rio de Janeiro, 2004, p. 241-261.

AZEVEDO, M. B. D. Efeito antinociceptivo da rilmenidina. 2002. 68 f. Dissertação (Mestrado em Fisiopatologia Clínica e Experimental) -
Universidade Estadual do Rio de Janeiro, Rio de Janeiro. 2002.

BRICCA, G.; DONTENWILL, M.; MOLINES, A. et al. Rilmenidine selectivity for imidazoline receptors in human brain. European Journal of Pharmacology, v. 163, p. 373-377, 1989.

BRONDANI, J. T.; NATALINI, C. C.; RAISER, A. G. et al. Analgesia epidural com clonidina ou romifidina em cães submetidos à cirurgia coxofemoral. Arquivo Brasileiro de Medicina Veterinária e Zootecnia, v. 56, n. 2. p. 175-182, 2004.

BROUX, B.; CLERCQ, D. D.; DE DECLOEDT, A. et al. Pharmacokinetics of intravenously and orally administered sotalol hydrochloride in horses and effects on surface electrocardiogram and left ventricular systolic function. The Veterinary Journal, 2015; DOI: http://dx.doi.org/10.1016/j.tvjl.2015.09.02 1.

BUTELMAN, E.R.; WOODS, J.H. Effects of clonidine, dexmedetomidine and xylazine on thermal antinociception in rhesus monkeys. Journal of Pharmacology and Experimental Therapeutics, v. 264, p.762-69, 1993. CARVALHO, A. M. A.; SOBRINHO, B. C.; SILVA, M. M. V. et al. Estudo comparativo dos efeitos cardiovasculares e sedativos da clonidina e xilazina como medicação pré-anestésica em cães. Revista Científica Eletrônica de Medicina Veterinária, ano 11, n. 20, 2013.

DÓRIA, R. G. S.; VALADÃO, C. A. A.; DUQUE, J. C. et al. Comparative study of epidural xylazine or clonidine in horses. Veterinary Anaesthesia and Analgesia, v. 35, p. 166-172, 2008.

FELDMAN, J.; TIBIRIÇÁ, E.; BRICCA, G. et al. Evidence for the involvement of imidazoline receptors in the central hypotensive effect of rilmenidine in the rabbit. British Journal of Pharmacology, v. 100, p. 600-604, 1990. 
GIOVANNONI, M. P.; GHELARDINI, C.; VERGELLI, C. et al. Alpha2-Agonists as Analgesic Agents. Medicinal Research Reviews, v. 29, n. 2, p. 339-368, 2009. GUIRRO, E. C. B. P.; SOBRINHO, G. R.; FERREIRA, I. M. M. et al. Efeitos comportamental, clínico e analgésico promovidos pela injeção epidural preventiva de morfina, xilazina ou clonidina, em equinos. Ciência Rural, Santa Maria, v. 41, n. 10, p. 1790-1796, 2011.

HAJHASHEMI, V.; MINAIYAN, M.; SEYEDABADI, M. Effect of tiazidine, rilmenidine, and yohimbine on naloxoninduced morphine withdrawal syndrome in mice. Iranian Journal of Pharmaceutical Research, v. 6, n. 2, p. 115-121, 2007.

KAMERLING, S. G.; CRAVENS, W. M. T.; BAGWELL, C. A. Objective assessment of detomidine-induced analgesia and sedation in the horse. European Journal of Pharmacology, v. 151 , p. $1-8,1988$.

KAMERLING, S. G.; WECKMAN, T. J.; DEQUICK, D. J. et al. A method for studying cutaneous pain perception and analgesia in horses. Journal of Pharmacological Methods, v. 13, p. 267-274, 1985.

OGATA, N.; DODMAN, N. H. The use of clonidina in the treatment of fear-based behavior problems in dogs: An open trial. Journal of Veterinary Behavior, v. 6, p. 130-137, 2011.

OLSON, M. E.; FIERHELLER, E.; BURWASH, L. et al. The efficacy of meloxicam oral suspension for controlling pain and inflammation after castration in horses. Journal of Equine Veterinary Science, v. 35; p. 724-730, 2015.

ONRAT, E.; KAYA, D.; KERPETEN, K. et al. Efectos de la rilmenidina en la función del sistema nervioso autónomo cardíaco de voluntarios sanos. Revista Española de Cardiologia, v. 57, n. 8, p. 745-750, 2004.
PACHALY, J. R. Terapêutica por Extrapolação Alométrica. In: CUBAS, Z. S.; SILVA, J. C. R.; CATÃO-DIAS, J. L. (Org.). Tratado de Animais Selvagens - Medicina Veterinária. 1 ed. São Paulo: Roca, 2006. p. 1215-1223.

REID, J. L. Rilmenidine: A clinical overview. The American Journal of Hypertension, v. 13, n. 6, p. 106-111, 2000.

ROMAN, A. A. S. R. Participação do Óxido Nítrico no Efeito Sedativo e Antinociceptivo dos Agonistas a2Adrenérgicos. 2004. 91 f. Tese (Doutorado em Patologia Experimental) - Departamento de Patologia Experimental, Universidade Federal Fluminense, Niterói. 2004.

SABETKASAIE, M.; KHANSEFID, N.; LADGEVARDI, A. R. S. Possible role of NMDA receptors in antinociception induced by rilmenidine in mice in the formalin test. European Journal of Pain, v. 11, p. 535-541, 2007.

SERRANO-RODRÍGUEZ, J. M.; GÓMEZ-DÍEZ, M.; CASTEJÓN-RIBER, C. et al. Pharmacokinetics and pharmacodynamics of ramipril and ramiprilat after intravenous and oral doses of ramipril in healthy horses. The Veterinary Journal; 2015; DOI: http://dx.doi.org/10.1016/j.tvjl.2015.10.02 4.

SPINOSA, H. S.; SPINOSA, F. R. N. Sobre os efeitos farmacológicos da xilazina. Biotemas, v. 4, n. 2, p. 111122, 1991.

STÄHLE, H. A historical perspective: Development of clonidine. Baillière's Clinical Anesthesiology, v. 14, n. 2, p. 237-246, 2000.

TIBIRIÇÁ, E.; FELDMAN, J.; MERMET, C. et al. Selectivity of rilmenidine for nucleus reticularis lateralis, a ventrolateral medullary structure containing imidazoline-preferring receptors. European Journal of Pharmacology, v. 209, p. 213-221, 1991. 